\title{
Looking for Molecular Changes in Blood of Cancer Patients after Applying IWCT. (A Scientific Hypothesis to Test)
}

\author{
Nooshin Abbasi ${ }^{1}$, Giordana Bettini² \\ 1 University of Padua \\ 2 University-Hos pital of Padova
}

\begin{abstract}
Cancer cells cause the number of white blood cells to reduce, weaken the immune system, and promote angiogenic factors to build special arterial networks or to coobtain blood, lymph and primo vessels that are already existed and allow them to make tumor microenvironment with a high quantity of specific nutrients and oxygen received from patient's blood and fluids; so that they are able to proliferate and spread from their primary site. In this study we aim to normalize tumor environment by extracting the tumorigenic nutrients from patient's blood, lymph and primo vascular system, starve cancer cells in where they were first formed, and boost immune system by assessing, for the first time, if Islamic Wet Cupping Therapy could clear the blood and body fluids from that specific substances and strengthen the immune system to let the body itself to fight against cancer.
\end{abstract}

\section{Definitions}

Nutrition and Growth

Defined by Mehdi Abbasi

Causative Pathological Substances (CPS)

Defined by Salah Mohamed El Sayed et al.

Taibah mechanism (Taibah Theory)

Defined by Salah Mohamed El-Sayed

Islamic Wet Cupping Therapy (IWCT)

Defined by Nooshin Abbasi

INTRODUCTION - Cancer cells are invaders that mislead, weaken the immune system, and drop the number of white blood cells (WBCs). Complicated arterial networks or even co-obtained blood, lymph and primo vessels from pre-existing ones feed cancer cells and 
provide them a friendly microenvironment where they can proliferate and spread to other parts of the body. ${ }^{[1]}$

The arterial network and blood, lymph and primo vessels enable them to receive nutrition from patient's blood and fluids. Cancer cells demand multiplex nutrients - such as acetate, glucose, glutamine, oleic acids, linolenic acid, short chain fatty acids: butyrate and propionate, FA beta-oxidation, lisolipids, plasma free amino acids (AAs): ornithine, glutamic acid and free tryptophan and inflammation signatures and etcetera - to divide and spread quickly. ${ }^{[2]}[3][4][5]$

\section{THIS ST UDY AIMS-}

1. To make changes in and normalize tumor microenvironment by extracting those additional multiplex nutrients demanded by cancer cells together with disease-related substances and send them away of blood and interstitial fluids based on their size differences, and

2. To boost immune system

both of these aims would be hoped to achieve simultaneously by applying Islamic Wet Cupping Therapy (IWCT) .

IWCT acts as a vacuum to create negative pressure through suction and it may cause the lighter hydrophobic and hydrophilic substances, the multiplex nutrients demanded by cancer cells, to separate from other blood and body fluids components and suck them out.

IWCT - also called Wet Cupping Therapy (WCT) is an old therapeutic technique to excrete waste or excess metabolic products from blood and interstitial fluids. In this technique a particular glass linked to a cupping pump creates a negative pressure to suck the viscoelastic skin in particular parts of the body specially in intrascapular area to raise blood volume, reduce pressure and to cause hyperemia thus increases capillary's filtration under the cup. It should be noted that the capillary networks with low blood velocity would be a point beside the primo vessels to deposit the harmful substances. The cup then is removed and a scalpel used to make small incisions on epidermis, the cup is replaced ag ain and suction is repeated several times to let blood and interstitial fluids drain from capillary networks with no returning to their venous end ${ }^{[6]}$. According to the Taibah mechanism presented in 2013 and based on scientific studies, this treatment showed desired results to improve different pathological conditions. ${ }^{[7][8][9][10][11]}$ 
Taibah mechanism illustrates the excretion that simulates renal glomerular filtration and abscess drainage. In this procedure a precutaneous excretion happens through skin capillaries in intrascapular area to clear blood and interstitial fluids from harmful substances or defined as causative pathological substances (CPS). ${ }^{[12]}$ Some studies showed the excretion of triglycerides low-density lipoprotein (LDL), cholesterol, total cholesterol, uric acid, inflammatory mediators, and immunog lobulin antibodies (rheumatoid factors), and also the reduction in pain severity, number of swollen joints and disease activity without any side effects after applying IWCT. [9][1 1 1][12] The intrascapular area where the cup will be usually positioned, on the brown adipose tissue (BAT) full of blood, lymph and primo vessels [13], is close to the sympathetic ganglia, the thoracic duct and the main vessels from heart and brain. ${ }^{[6]}$ The Primo Vascular System (PVS) surrounds and is connected to tumor tissue and the primo fluid is a more effective place for tumor cells to circulate in body. ${ }^{[13]}$ Some studies suggest the PVS as a potential metastasis rout beside lymphatic and hematologic systems. ${ }^{[14]}[15]$ According to the pioneering work of Dr. William Coley and his daughter, Dr. Helen Coley Nauts, on cancer immunotherapy presented in 1980s, the second target of this study (as mentioned briefly) while trying to get rid of tumorigenic nutrients and disease-related substances or CPS from patient's blood and fluids follows the stimulating innate immune system via size-dependent and pressure-dependent stress of IWCT. IWCT improves the natural immunity and decrease auto-antibodies, inflammatory mediators, and ferritin in serum. ${ }^{[12]}$

MET HODS- This study is going to, for the first time, analyze the Venus blood of patients before and after applying IWCT together with the cupping blood itself to see if this therapeutic technique may make any molecular changes in and normalize blood of cancer patients to support the giving predictions.

So far all the analyses and scientific studies on wet cupping therapy in different pathological conditions but not in cancer have compared the Venus blood sample before applying wet cupping therapy with cupping blood. But the problem with this comparison would be that because of suction the analyzed factors are obviously more concentrated in cupping blood rather than in Venus blood so this comparison won't give a clear result on what positive changes this technique may make into the blood.

We are going to apply the mentioned technique, for the first time, on cancer patients and 
to not just compare the Venus blood with cupping blood but also the Venus blood before IWCT with Venus blood after IWCT that can hopefully confirm whether the positive changes in blood have truly occurred if any.

To doing so it should be studied:

1. If IWCT could create circulatory shear stress that breaks the built tolerance between host and tumor and adapts immune responses to provoke anti-tumor effects; these effects may include the production of Natural Killers (NKs) and decreasing cytokines interleukins 6/8 that prevent circulating tumor cells (CTCs) from metastasis, ${ }^{[16]}$

2. If IWCT is also able to make any changes in expression of Plakoglobin as the adhesion protein that keeps the CTCs' cluster, ${ }^{[16][17]}$

3. If IWCT affects the CTCs' mitochondrial activity and if it is able to decrease mitochondrial/ oxidative phosphorylation as this phosphorylation is essential for cancer cells motility. ${ }^{[18]}$

4. In addition, pressure-dependent effects of IWCT may be able to take out CTCS, which can be found in the white blood cells (WBCS) fraction and in PVS. CT Cs are generally larger than WBCs ${ }^{[19]}$. The suction created in IWCT may be able to capture and take the CT CS out of blood and body fluids.

5. Different cancer biomarkers and molecules should be also tested to assess any possible alteration of these factors in blood and body fluids of cancer patients after applying IWCT .

CONCLUSION- Considering Islamic Wet Cupping Therapy as a scientific method and test it as a new procedure to fight against cancer may lead us to target the tumor microenvironment and hunger cancer cells in their primary place and promote the immune system. According to this hypothesis the cancer cells then become starved and could be easily destroyed by a strong immune system in a microenvironment that is not anymore belonging to them.

\section{References}

1. ^Nooshin Abbasi. Could the General Law of Nutrition \& Growth Be also Applicable to Cancer Cells' Growth?

2. ^ AlexanderJ. Lakhter, James Hamilton, Raymond L. Konger, Nickolay Brustovetsky, HalE. Broxmeyer, Samisubbu R. Naidu. (2016). Glucose-independent Acetate Metabolism Promotes Melanoma Cell Survival and Tumor GrowthJ. Biol. Chem., vol. 
291 (42), 21869-21879. doi:10.1074/jbc.m115.712166

3. ^Jie Shen et al.. (2013). Plasma Metabolomic Profiles in Breast Cancer Patients and Healthy Controls: By Race and Tumor Receptor Subtypes Translational Oncology, vol. 6

4. ^ Cascino A. et al.. (1995). Plasma amino acid imbalance in patients with lung and breast cancer Anticancer Research

5. ^ Zachary T. Schug, Barrie Peck, Dylan T. Jones, Qifeng Zhang, Shaun Grosskurth, Israt S. Alam. (2015). Acetyl-CoA Synthetase 2 Promotes Acetate Utilization and Maintains Cancer Cell Growth under Metabolic Stress Cancer Cell, vol. 27 (1), 57-71. doi:10.1016/j.ccell.2014.12.002

6. a,b Roshanak Ghods, Nasrin Sayfouri, Mohammad Hossein Ayati. (2016). Anatomical Features of the Interscapular Area Where Wet Cupping Therapy Is Done and Its Possible Relation to Acupuncture Meridians. Journal of Acupuncture and Meridian Studies, vol. 9 (6), 290-296. doi:10.1016/j.jams.2016.06.004

7. ^ El Sayed SM Mahmoud HS. (2013). Medical and Scientific Bases of Wet Cupping Therapy (Al-hijamah): in Light of Modern Medicine and Prophetic Medicine Altern Integ Med, vol. 02 (05) doi:10.4172/2327-5162.1000122

8. ^ Huijuan Cao, Xun Li, Jianping Liu. (2012). An Updated Review of the Efficacy of Cupping Therapy PLoS ONE, vol. 7 (2), e31793. doi:10.1371/journal.pone.0031793

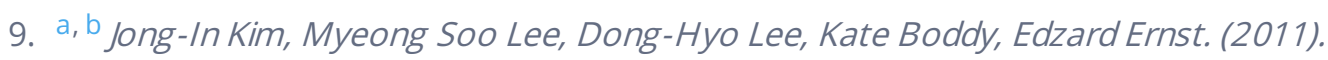
Cupping for Treating Pain: A Systematic Review Evidence-Based Complementary and Alternative Medicine, vol. 2011 , 1-7. doi:10.1093/ecam/nep035

10. ^ Huijuan Cao, Mei Han, Xun Li, Shangjuan Dong, Yongmei Shang, Qian Wang. (2010). Clinical research evidence of cupping therapy in China: a systematic literature review BMC Complement Altern Med, vol. 10 (1) doi:10.1186/1472-6882-10-70

11. a, b Salah El Sayed, Ashraf Abou-Taleb, Manal Mohaed Nabo, Hany Mahmoud, Nagwa Ahmed, Hussam Baghdadi. (2014). Al-hijamah and oral honey for treating thalassemia, conditions of iron overload, and hyperferremia: toward improving the therapeutic outcomes JBM doi:10.2147/jbm.s65042

12. a, b, c Hussam Baghdadi et al. (2015). Ameliorating Role Exerted by Al- Hijamah in Autoimmune Diseases: Effect on Serum Autoantibodies and Inflammatory Mediators International Journal Of Health Sciences, vol. 9

13. a, b Bruno Chikly, Paul Roberts, Jörgen Quaghebeur. (2016). Primo Vascular System: A Unique Biological System Shifting a Medical Paradigm.J Am Osteopath Assoc, vol. 116 (1), 12. doi:10.7556/jaoa.2016.002 
14. `Jung Sun Yoo, Hong Bae Kim, Nayoun Won, Jiwon Bang, Sungjee Kim, Saeyoung Ahn. (2010). Evidence for an Additional Metastatic Route: In Vivo Imaging of Cancer Cells in the Primo-Vascular System Around Tumors and Organs. Mol Imaging Biol, vol. 13 (3), 471-480. doi:10.1007/s11307-010-0366-1

15. ^ Kwang-Sup Soh. (2015). Hypothesis on the Treatment of Gliomas with Acupuncture at the Primo Node Corresponding toZusanli(ST 36). Medical Acupuncture, vol. 27 (3), 144-150. doi:10.1089/acu.2014.1089

16. ${ }^{a}, \mathrm{~b}$ K Pantel, M R Speicher. (2015). The biology of circulating tumor cells Oncogene, vol. 35 (10), 1216-1224. doi:10.1038/onc.2015.192

17. ^ Lingeng Lu, HongmeiZeng, Xinsheng Gu, Wenxue Ma. (2015). Circulating tumor cell clusters-associated gene plakoglobin and breast cancer survival Breast Cancer Res Treat, vol. 151 (3), 491-500. doi:10.1007/s10549-015-3416-1

18. ^ Valerie S. LeBleu, Joyce T. O'Connell, Karina N. Gonzalez Herrera, Harriet Wikman, Klaus Pantel, Marcia C. Haigis. (2014). PGC-1 $\alpha$ mediates mitochondrial biogenesis and oxidative phosphorylation in cancer cells to promote metastasis Nat Cell Biol, vol. 16 (10), 992-1003. doi:10.1038/ncb3039

19. ^ S. C. P. Williams. (2013). Circulating tumor cells Proceedings of the National Academy of Sciences, vol. 110 (13), 4861-4861. doi:10.1073/pnas.1304186110 